Thérapie familiale, Genève, 2005, Vol. 26, No 1, pp. 19-35

\title{
UNE MODÉLISATION DE LA COMMUNAUTÉ RIGIDE
}

\author{
La famille d'origine du père d'Anne
}

\section{E. DESSOY ${ }^{1}$, M. STASSART ${ }^{2}$, A. COURTOIS ${ }^{3}$, G. BERNAERTS ${ }^{4}$, A. DE KEYSERS ${ }^{5}$,

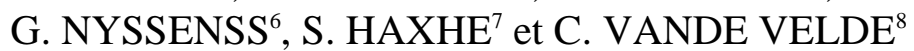

Résumé : Une modélisation de la communauté rigide la famille d'origine du père d'Anne. Cet article présente une modélisation du clan dont le principe organisateur est un « antagonisme caché ». Les croyances familiales unissent l'ensemble des membres autour d'un projet commun lié au paraître et à l'avoir. Mais un sous-système se construit, qui réintroduit une différence dans ce monolithe, à partir de valeurs proches de l'être et de l'éprouvé, des valeurs liées à l'ambiance, à la spiritualité, à l'esthétique, à la souffrance et à la pathologie. Ce sous-système appelé «contestant caché », dissimulé dans le maquis familial, n'est reconnu par personne, et pourtant il agit avec autant de puissance que

1 .Professeur de psychologie clinique aux Facultés de Psychologie et des Sciences de l'Education des Universités de Louvain-la-Neuve et de Liège. Psychothérapeute et formateur en thérapie familiale et en intervention systémique au centre «Chapelle-aux-Champs » et à l'IFISAM à Bruxelles.

2. Chef de travaux en Psychologie clinique au Département «Personne et Société» de la Faculté de Psychologie et des Sciences de l'Education de l'Université de Liège. Psychothérapeute.

3.Chargée de cours invitée à l'Unité de Psychologie clinique «Anthropologie, Psychopathologie et Psychothérapie» de la Faculté de psychologie et des Sciences de l'Education de l'Université de Louvain-laNeuve. Maître de conférence à l'Université de Liège. Psychothérapeute.

4. Assistante en psychologie clinique au Département «Personne et Société» de la Faculté de psychologie et des Sciences de l'Education de l'Université de Liège. Psychothérapeute.

5. Assistantes à l'Unité de Psychologie clinique « Anthropologie, Psychopathologie et Psychothérapie » de la Faculté de Psychologie et des Sciences de l'Education de l'Université de Louvain-la-Neuve.

Psychothérapeute.

6. Assistante au Département «Personne et Société» de la Faculté de Psychologie et des Sciences de l'Education de l'Université de Liège.

7. Assistante à l'Unité de Psychologie clinique «Anthropologie, Psychopathologie et Psychothérapie» de la Faculté de Psychologie et des Sciences de l'Education de l'Université de Louvain-la-Neuve. 
l'autre sous-système appelé «dominant», pour soutenir l'unité de la communauté, mais par des moyens radicalement opposés à ceux du sous-système «dominant».

Le processus organisateur du clan est donc animé par un antagonisme caché entre deux sous-systèmes dont chacun tente d'imposer ses propres moyens de produire l'unité, alors que le clan se nourrit de leur lutte sous-jacente.

Summary : A modeling of the rigid community. Family of origin of Anne's father. - This paper is a modeling of the rigid community or clan which the organizing core is a «hidden antagonism». The family beliefs cluster the members around a joint project related to appearing and to having it. But a subsystem develops and reintroduces a difference in this monolith. This subsystem is based on elements related to being and feeling, but also to ambiance, spirituality, aesthetics, suffering and pathology. This « hidden opposing » force dissimulated in the family « underground » remains unknown but works with an equal power to sustain the community unity. However, it uses radically different means than the dominating subsystem. Although each subsystem thinks it supports the system unity, it is more the conjunction of the two of them (in a hidden antagonism) that is the organizing principle of the clan.

Resumen : Una modelizaciôn de la comunidad rigida. La familia de origen del padre de Anne. - Este articulo presenta una modelizaciôn del clan cuyo principio organizador es un «antagonismo escondido ». Las creencias familiares unen al conjunto de los miembros alrededor de un proyecto comôn vinculado al parecer y al tener. Pero, un subsistema quién reintroduce una diferencia en este monolitico se construye a partir de valores prôximos del ser y del sentir, valores vinculados al ambiente, a la espiritualidad, a la estética, al sufrimiento y a la patologfa. Este subsistema llamado «respondedor escondido », disimulado en la organizaciôn familiar, no es reconocido por nadie y sin embargo acutna con tanta fuerza como et otro subsistema, llamado «dominante », para sostenir la unidad de la comunidad. La diferencia est en que los medios utilizados por cada uno de estos sub-sistemas son radicalmente opuestos. El proceso organizador del clan es entonces animado por un antagonismo escondido entre estos dos subsistemas que intentan imponer sus propios medios de crear la unidad. El clan se nutre asi de su lucha subyacente.

Mots-clés: Clan familial - Antagonisme organisateur - Mythe - Double lien - Triple contrainte.

Keywords : Family clan - Organizing antagonism - Myth - Double bind - Triple bind.

Palabras claves : Clan familiar - Antagonismo organizador - Mito - Doble vinculo Triple vfnculo.

En 1997, la revue Thérapie Familiale publie notre article « Ambiance et double lien » (5) et l'année suivante «Croyances et double lien » (6). Ces deux textes n'avaient qu'un but, mettre en évidence l'impact du double lien sur les foyers organisateurs du milieu humain (3), d'abord sur l'ambiance et l'éthique ${ }^{9}$, ensuite sur les croyances, en développant l'idée

\footnotetext{
${ }^{9} \mathrm{Si}$ les notions de croyances et d'ambiance se comprennent aisément, celle d'éthique risque de prêter à
} 
de G. Bateson selon laquelle les doubles contraintes ne sont pas des phénomènes isolés «parce qu'elles renvoient à la structure contextuelle totale, si bien qu'un exemple donné de double contrainte repéré pendant une séance de thérapie ne sera que le sommet d'un iceberg qui aura pour structure fondamentale l'ensemble de la vie familiale ${ }^{10} \gg$. Notre recherche du contexte du double lien, à l'aide de l'idée de milieu, a effectivement permis d'élargir le champ du double lien à la dimension de la famille nucléaire en explicitant l'intuition de G. Bateson. Avant de poursuivre la recherche, en direction cette fois des familles d'origine du père et de la mère d'une jeune fille que nous appellerons Anne, nous allons rappeler la problématique de la famille nucléaire dont le modèle de la triple contrainte permet de décrire l'organisation.

\section{La triple contrainte}

L'article «Ambiance et double lien » (5) décrit d'abord la rencontre d'une mère, Jeanne, et de sa fille, Anne, une adolescente de 15 ans, lors d'une première consultation thérapeutique demandée pour Anne. Selon la mère, sa fille ne se développe pas normalement, elle reste infantile, manque d'initiative et reste constamment accrochée à

confusion. Le plus souvent, en effet, le mot s'écrit avec une majuscule, et l'Ethique se réfère alors à une tradition qui englobe un ensemble de réalités que, pour notre part, nous situons dans les croyances, par exemple, les grandes valeurs humaines. Beaucoup de philosophes considèrent qu'il existe une Ethique à laquelle l'humanité entière aurait intérêt à se conformer. En nous appuyant sur d'autres auteurs, l'éthique est pour nous une réalité plus modeste synonyme de mise en acte, d'actions concrètes qui se mettent en scène dans un milieu déterminé et dont les croyances et les représentations de ce milieu en sont la justification. Dès lors, nous pensons que chaque milieu humain, chaque famille, chaque institution possède sa propre éthique constituée par les normes, les règles concrètes et les rites que chacun d'eux met en scène grâce à la communication et en particulier l'interaction si bien décrite par la «pragmatique de la communication ». Les normes, les règles et les rites se réfèrent au système de croyances d'un milieu particulier et ils infléchissent son ambiance. Plutôt donc que de considérer l'Ethique comme une réalité absolue et universelle, nous considérons l'éthique comme une réalité relative à chaque milieu particulier. L'intérêt d'une telle conception réside dans le fait qu'à présent, il est possible d'aller à la recherche du bien-fondé des comportements (mise en scène) d'une famille qui, par exemple, se montre violente, sans pour autant poser un jugement de valeur sur elle, jugement qui reposerait sur une Ethique supposée universelle. Il semble que cette approche offre au thérapeute un grand degré de liberté dans son essai de comprendre ce qui est en «cause» dans une perspective systémique. Les situations sont nombreuses qui montrent une incompréhension totale de certaines personnes confrontées, par exemple, à la Justice et qui sont condamnées pour fait de violence sur un enfant, alors que chez eux, mais aussi souvent dans le quartier qu'ils habitent, la correction physique est un acte éthique et valorisé. Le propos n'est évidemment pas de protéger de telles pratiques mais de permettre au thérapeute d'accéder à la véritable culture de ces gens afin d'établir un dialogue entre leur éthique reconnue et l'Ethique de la société dominante à laquelle nous, thérapeutes, appartenons en bonne partie.

${ }^{10}$ Voici un extrait de La Peur des Anges, un livre écrit par Bateson et sa fille Mary Catherine (1) Le père: « [...] Regarde l'horrible affaire de la thérapie familiale, avec tous ces thérapeutes qui font des "interventions paradoxales" pour changer les individus ou les familles, ou qui comptent les "doubles contraintes". Les doubles contraintes ne se comptent pas. »

La fille: «Non, parce qu'elles renvoient à la structure contextuelle totale, si bien qu'un exemple donné de double contrainte repérée pendant une séance de thérapie ne sera que le sommet d'un iceberg qui aura pour structure fondamentale l'ensemble de la vie familiale. Mais tu n'empêcheras pas les gens d'essayer de compter les doubles contraintes, car la propension à décomposer les processus en un certain nombre d'entités est une composante essentielle de la perception humaine [...] ». 
ses basques. En fait, la mère enjoint à sa fille d'être libre et autonome. Mais tout en tenant compte de ce discours déjà paradoxal, Jeanne se comporte avec l'adolescente comme si elle était une petite enfant qu'elle ne cesse de protéger et de manipuler tout au long de la séance. Le double lien semble être le concept le plus adéquat pour qualifier cet ensemble de communications qui ligote les deux femmes. Anne est une enfant unique et son père est invité à la deuxième séance.

Selon les spécialistes qui avaient déjà été consultés, Anne souffre d'un trouble psychique endogène. En effet, les modifications du contact qu'elle éprouve confirment l'hypothèse d'un tel trouble: Anne souffre d'une perturbation majeure du contact qui correspond effectivement à une pathologie liée à la santé mentale. Cette perturbation étant en conjonction directe avec le double lien, il est dorénavant légitime de poser l'existence d'un lien intrinsèque entre la maladie d'Anne et le double lien ${ }^{11}$. Mais la nature exclusivement endogène de la maladie est mise en question par cette même légitimité: d'une part, les perturbations du contact s'inscrivent en même temps dans l'ambiance familiale et, d'autre part, le double lien est un processus interactif qui associe plusieurs acteurs et, en cela, il concerne directement l'éthique familiale. L'ambiance et l'éthique de la famille prennent donc part à la production et au maintien du problème d'Anne, faisant de sa maladie un phénomène qui s'inscrit aussi dans le milieu familial en associant le caractère endogène de la maladie à un caractère exogène, son milieu d'appartenance. L'article montre donc que l'inscription (bio)-psychologique de la maladie s'associe étroitement à une inscription sociétale et qu'ensemble, ces deux inscriptions maintiennent le problème. Antérieurement, nous avions déjà montré, d'une première manière, l'importance d'un trouble du contact et d'une perturbation de l'ambiance dans l'avènement d'une pathologie psychotique chez l'enfant (4). Il est dorénavant possible d'affirmer que ne pas tenir compte de l'impact du double lien sur l'ambiance est se condamner à ne rien comprendre, entre autres, dans les manifestations du psychotique et de l'autiste. De même, en ce qui concerne le toxicomane, le trouble majeur du contact qu'il éprouve est en rapport direct avec le double lien : ce trouble consiste à subir constamment une rupture du contact que le toxicomane comble par le produit consommé. En quelque sorte, la drogue et l'alcool sont déjà des «produits de substitution ». Notre réflexion permet ainsi d'infléchir la perspective thérapeutique en reconsidérant l'importance de l'ambiance dans le traitement d'une gamme étendue de pathologies.

L'impact du double lien sur l'éthique (cf. note 8) n'a pas fait l'objet d'une discussion spécifique tant son influence est évidente. Nous n'avons cessé de souligner que sa double structure culturelle - le code normatif et les comportements analogiques qui expriment le style - est totalement clivée par le double lien. Le code normatif est en parfaite accointance avec les croyances sous-tendues par le seul espace distancié : "Mais quand vas-tu, enfin, te comporter comme une grande fille ! », dit la mère, tandis que les comportements analogiques dénient ce message : en deçà des mots, les comportements de la mère et d'Anne mettent en scène une mère à la dévotion d'une petite fille incapable

${ }^{11}$ Les pragmaticiens n'ont pas établi une relation directe entre ce type de double lien et l'émergence d'une maladie mentale. Par prudence, ils ont dit que l'on remarque souvent l'émergence d'une maladie conjointement à l'existence d'un double lien chronique. Mais en enrichissant la réflexion, à partir de l'ambiance, un psychanalyste szondien, par exemple, déterminera aisément un trouble majeur du contact qu'il liera directement à un trouble de la personnalité. 
encore de s'occuper d'elle-même. Anne se comporte comme un enfant, ce qui justifie et renforce les comportements de la mère. Alors qu'une situation dynamique voit le code normatif et les comportements analogiques tenter de manifester plus ou moins la même éthique, dans une situation de double lien, ces deux réalités sont clivées au point d'être incompatibles. Si donc la littérature concernant le double lien a surtout souligné l'incompatibilité entre deux modes de communication (relevée dans l'injonction paradoxale ou la définition paradoxale), lorsque nous examinons l'impact du double lien dans l'éthique de la communauté, nous découvrons un autre clivage, cette fois, au niveau des normes culturelles.

L'article «Croyances et double lien » (6) poursuit la réflexion en direction des croyances familiales et de l'impact du double lien sur elles. Alors que l'investigation de l'ambiance laisse intactes les rumeurs d'une «mère schizophrénogène», sorte de suppôt de Satan responsable des malheurs de sa fille, l'abord des croyances rend obsolète cette accusation, car une nouvelle figure s'impose, celle du père « absent», figure insaisissable mais très souvent rencontrée dans la clinique, et dont Lacan parle en terme de forclusion. Ce père « absent », nous l'avons convoqué en lui reconnaissant une place tout aussi importante que celle de la mère dans la production et le maintien du double lien.

La fonction qu'il prend, d'imposer le seul espace distancié à l'ensemble de la famille qui accepte sans rien dire, s'apparente à un paradoxe de type logique dans la mesure où la famille situe le père à deux niveaux : il est à la fois conjoint et à ce titre au même niveau logique que sa femme et, en même temps, à un niveau logique supérieur, celui où se situe la loi. En effet, si la famille accepte tacitement la loi de distanciation à une telle intensité, tout se passe comme si le père incarnait lui-même cette loi, alors que celle-ci est un phénomène qui émerge d'une perpétuelle négociation des membres de la famille et, à ce titre, qui se situe à un niveau logique supérieur à celui des personnes.

Ce pseudo accord dissimule une antinomie dans les croyances des conjoints qui se traduit par un divorce émotionnel que le couple éprouve constamment sans jamais l'exprimer. Si le père exprimait la représentation qu'il a d'un couple, il y attacherait des valeurs exclusivement liées à la liberté, à l'autonomie et au développement personnel de chacun, tandis que la mère soutiendrait des valeurs inverses où l'unité familiale et la solidarité seraient à l'avant-plan. Si ces deux énoncés étaient mis bout à bout, la phrase répondrait à la définition d'une antinomie sémantique. Le vide émotionnel que ressent la mère est insupportable car elle doit l'accepter activement et en même temps dénier le vide et la souffrance qu'elle ressent.

C'est alors qu'elle invente un nouveau mode de relation avec sa fille - le double lien - qui respecte l'espace distancié, tout en créant un puissant sous-système unitaire. Son discours : «Je veux que tu sois autonome et que tu te prennes en main » est soustendu par un espace distancié ; la forte proximité entre elle et sa fille se repère uniquement dans les comportements, tandis qu'un espace distancié est principalement vécu dans l'ambiance. Même dans le double lien, la mère respecte donc « l'ordre » du père, la distance, et celuici ne peut reprocher à sa femme la proximité comportementale puisqu'elle est imposée par les agissements symptomatiques d'Anne.

La soudure organisationnelle de l'ensemble se réalise par un «antagonisme caché », dissimulé par l'organisation particulière de la famille : les deux antagonistes premiers sont les époux. Par la suite, leur antagonisme se dissimule lors de l'avènement du patient désigné qui développe un autre antagonisme en direction du père, un antagonisme très 
particulier qui ne se manifeste pas au premier abord. Cette dissimulation en cascade des antagonismes situe donc le patient désigné comme tiers d'un désaccord parental dont nous allons poursuivre l'étude. Nous avons appelé « triple contrainte » l'ensemble du processus opérant la soudure organisationnelle de la famille nucléaire, parce qu'il associe les trois types de paradoxes rencontrés dans la nature"12 : à partir d'un divorce émotionnel qui ne peut se résoudre et qui prend figure d'antinomie ( $1 \mathrm{er}$ paradoxe), le père et ses alliés initient un paradoxe logicomathématique (2e paradoxe) à quoi répond le double lien (3e paradoxe) qui, à son tour, renforce le paradoxe initié par le père, etc.

Ainsi, il y a six ans, se terminait provisoirement l'étude du double lien resitué dans le contexte d'une famille nucléaire: il suscite une modification radicale des trois foyers organisateurs du milieu humain laissant déjà apparaître une partie de la structure subaquatique de l'iceberg dont parle G. Bateson.

L'approche thérapeutique du double lien a produit une littérature non négligeable, mais finalement peu convaincante tant elle s'est limitée quasi au seul niveau comportemental. Resitué dans une triple contrainte, on comprend aisément que le double lien n'est pas qu'un parasite communicationnel à éliminer, il est un des trois moteurs d'un processus plus large qui inclut un divorce émotionnel dans le couple parental et la fonction occultée du père et de ses alliés. Dès lors, vouloir se centrer sur le seul double lien dans l'espoir de l'éradiquer est généralement peine perdue". L'introduction de la fonction du père apportet-elle de nouveaux éléments susceptibles d'intéresser le thérapeute? Elle l'éclaire sur une complexité accrue du problème d'Anne, mais il est tenté alors de détourner son attention de la mère pour s'intéresser au père en voulant l'aider à réduire la distance qu'il impose. Là encore, le thérapeute risque fort de vivre l'échec dans la mesure où il touche de nouveau à un processus partiel qui prend sens dans un contexte plus large. Au point où nous en sommes, il est vrai que nous n'accédons encore ni au sens des phénomènes que nous avons décrits, ni à leur légitimité à partir de quoi nous pourrions penser un traitement. Quelles sont les forces qui poussent le père à se comporter comme il le fait? Qu'estce qui incite la mère à se taire et à voiler ce divorce émotionnel? Qu'est-ce qui l'incite souvent à soutenir activement son mari? Nous ne sommes pas encore en mesure de répondre à ces questions ; c'est pourquoi nous investiguerons tour à tour les familles d'origine des deux parents d'Anne, en commençant par la famille du père, à la recherche de réponses à partir desquelles une nouvelle forme de traitement pourrait se construire.

\section{La place possible du père dans sa famille d'origine}

Nous avons choisi, parmi les multiples modélisations possibles de la famille d'origine du père d'Anne, celle que nous avons rencontrée le plus souvent dans notre clinique : une communauté soudée par des liens particuliers que nous décrirons au fil du texte. Qu'il soit bien entendu que toutes les situations de «père absent»n'ont pas nécessairement leurs

${ }^{12}$.P. Watzlawick, dans son ouvrage « Une logique de la communication », aux pages 190 et 191, avance l'idée que «les paradoxes pragmatiques naissent pour ainsi dire des deux autres types»; les coauteurs de l'ouvrage avaient le ferme projet de développer la question, mais ils se sont contentés d'en rester à l'étude du paradoxe pragmatique et de développer, par conséquent, une forme de thérapie aux allures comportementales. 
origines dans le modèle que nous allons présenter.

Quant à la manière de présenter ce modèle, nous avons hésité. Eut-il été préférable d'exposer une étude de cas dans son intégralité qui aurait souligné avantageusement l'originalité d'une famille construisant une configuration que nous appelons modèle ? Cette procédure ne permet cependant pas de mettre en évidence les variations possibles

du modèle, et nous ne l'avons pas retenue, préférant choisir, à partir des nombreuses situations cliniques rencontrées, quatre études brèves qui montrent progressivement la complexité du modèle et de ses enjeux. Certaines apparaîtront au lecteur comme une reconstruction "didactique », elles proviennent néanmoins toutes de notre expérience clinique. Dans ce qui suit, le lecteur découvrira donc quatre pères hypothétiques qu'Anne aurait pu avoir.

\section{Le soutien de veuve (figure 1)}

Une première manière de comprendre la distance émotionnelle et parfois physique que le père impose à sa famille nucléaire consiste à choisir, parmi les nombreux cas de figure possibles, celui où il est simplement soutien de veuve. Enfant unique, son père est mort depuis longtemps, il est la seule personne sur laquelle sa vieille mère peut s'appuyer. Représentant en produits pharmaceutiques, il programme son travail de telle façon qu'il puisse manger à midi presque chaque jour avec sa mère. A l'entendre, il l'aide à faire ses courses et à tenir son ménage, il est aux petits soins auprès d'elle et veille à ce qu'elle ne manque de rien. La forte complémentarité qui soude la grand-mère et le père d'Anne contraste avec la distance qu'il semble imposer dans la famille nucléaire. Cette situation fait penser à la " figure de Janus », d'un côté le père est un homme de distance, de l'autre un homme de grande proximité. La capacité d'activer des espaces opposés montre que l'espace n'est pas «inscrit dans les gènes » d'une personne, mais plutôt que celle-ci est capable d'activer des espaces circonstanciels liés à des contextes différents. Au cours du traitement, nous avons suggéré au père de mettre de la distance avec sa mère, mais sa femme a réagi la première en expliquant combien la chose est impossible. Le couple est convaincu que la grand-mère mourrait si son fils cessait de l'entourer.

Il est aisé de comprendre le lien entre l'implication du père vis-à-vis de sa mère et la distance qu'il impose dans sa famille nucléaire: s'il veut témoigner une grande fidélité à sa mère, il ne peut s'impliquer tout autant dans sa famille nucléaire et tout se passe 


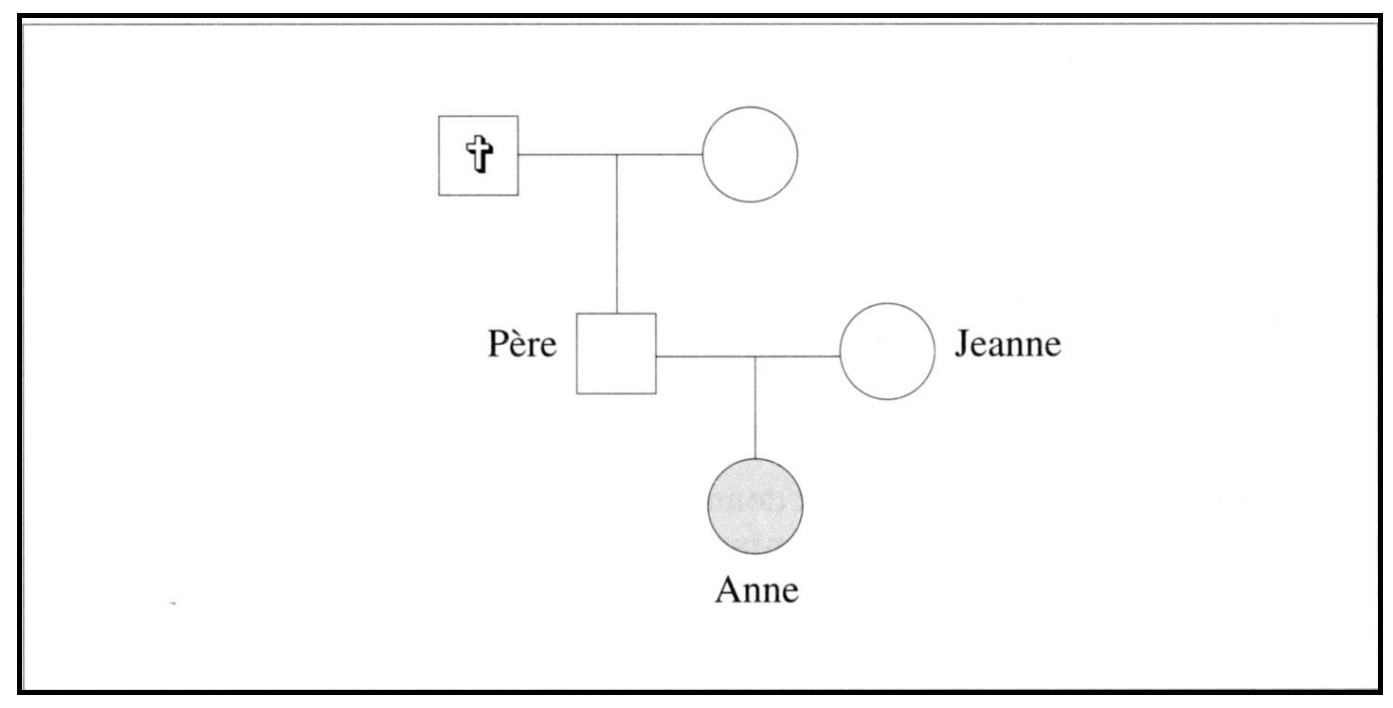

Figure 1: Famille du père d'Anne dans la configuration 1. Soutien de de veuve.

comme s'il vivait un conflit de fidélité vis-à-vis de deux femmes. Il est néanmoins curieux que son épouse ne se montre pas davantage jalouse, c'est même elle qui veut maintenir la situation quand nous proposons au père de mettre un peu de distance entre lui et sa mère.

Lorsque le problème d'Anne nous apparut à ce point lié à la famille d'origine du père, nous avons demandé au couple d'inviter la grand-mère paternelle à la prochaine séance. Après quelques hésitations, le couple a accepté et le jour dit, déjà dans la salle d'attente, la grand-mère nous a pris à part et nous a dit : «Ne trouvez-vous pas que mon fils est trop dépendant de moi ? ». C'était une grande femme, bien bâtie, en bonne santé, qui est entrée de pied ferme dans la salle de réunion.

La séance fut éprouvante pour le père car la grand-mère, en faisant alliance avec le thérapeute, en le prenant à témoin, a exprimé sa désolation vis-à-vis de l'attitude si infantile et dépendante de son fils qui ne pouvait vivre sans elle. Elle étayait son discours d'exemples tels que celui-ci : «Souvent mes amies profitent de voyages organisés, elles passent un week-end à Paris, trois jours à Londres, etc. Cela fait des années que je me prive de voyager avec elles parce qu'il faut que j'accueille mon fils et que je lui fasse à manger! ». Le père pâlissait au fur et à mesure que sa mère parlait. Lui et sa femme se rendaient compte de l'immense quiproquo qu'ils avaient vécu durant tant d'années alors qu'il avait tout fait pour adoucir la vie de sa vieille mère : tandis que le couple était persuadé que la grand-mère ne survivrait pas si son fils cessait de l'entourer, la grandmère exprimait sa plainte de sacrifier sa vie à s'occuper encore de son fils « incapable de vivre sans elle ». Il fallut plusieurs séances pour que le père « encaisse » cette nouvelle qui bouleversait la représentation qu'il avait de sa mère et du rôle qu'il avait à remplir auprès d'elle.

Après cet épisode, le père tenta de distancier ses liens avec sa mère et il se tourna davantage vers sa femme qu'il semblait découvrir sous un nouveau jour. C'était la réaction que nous attendions, une attitude propice à transformer le divorce émotionnel du couple en un nouveau «contrat de mariage », une nouvelle organisation de la famille nucléaire qui libérerait Anne et sa mère du double lien dans lequel elles étaient maintenues prisonnières. Mais, contre toute attente, la femme n'a pas ouvert ses bras à 
son époux retrouvé, elle a réagi aux propositions de son mari en augmentant elle-même la distance, comme si elle voulait réaffirmer le divorce émotionnel. Nous avons dû nous rendre à l'évidence que l'épouse, elle aussi, avait de bonnes raisons de maintenir une forte distance dans son couple. Nous ne pouvions donc pas faire l'économie d'un travail avec sa famille d'origine, il nous restait à comprendre, d'une part, comment son comportement avait été appris dans le passé et, d'autre part, la fonction qu'elle occupait encore dans sa famille d'origine.

\section{Deux frères en antagonisme : une soudure communautaire (figure 2)}

Continuons notre construction et donnons un frère aîné au père d'Anne. Ce frère est ingénieur, marié et père de deux enfants en excellente santé. Il vit avec sa famille en Suède et revient très rarement en Belgique ; à Noël et aux grandes vacances, il passe en coup de vent avant de se rendre dans le sud de la France. Sur la cheminée de leur mère, seules des photos du frère aîné et de sa famille, sauf, dans le coin, une photo d'Anne quand elle était petite et qu'elle souriait encore. Le frère aîné «a bien réussi », tandis que le père d'Anne a échoué en médecine et s'est retrouvé délégué commercial en produits pharmaceutiques. Aux yeux de la famille, c'est un raté. La grand-mère, les oncles et les tantes ne cessent de louanger le frère aîné, d'être fiers de lui, tandis qu'on ne dit mot du frère cadet. Pourtant, chaque jour, il est près de sa mère, à son service, et il adoucit ses vieux jours, tout en l'écoutant parler de son grand frère et de sa brillante réussite professionnelle et familiale. Les deux frères ne s'adorent guère et pourtant, enfants et adolescents, ils jouaient volontiers ensemble; mais la mort du père et l'échec universitaire du cadet ont créé une certaine distance entre eux. La grand-mère et le fils cadet insistent néanmoins sur le fait qu'ils forment une famille très unie; c'est la face visible, une image d'Epinal ou un mythe familial. Car en énonçant des phrases conviviales sur sa famille, le père d'Anne soupire souvent ou montre des signes d'agacement, voire parfois de l'opposition. Cette famille très soudée évite de se différencier, seule l'unité de la communauté est l'objet de leur discours. 


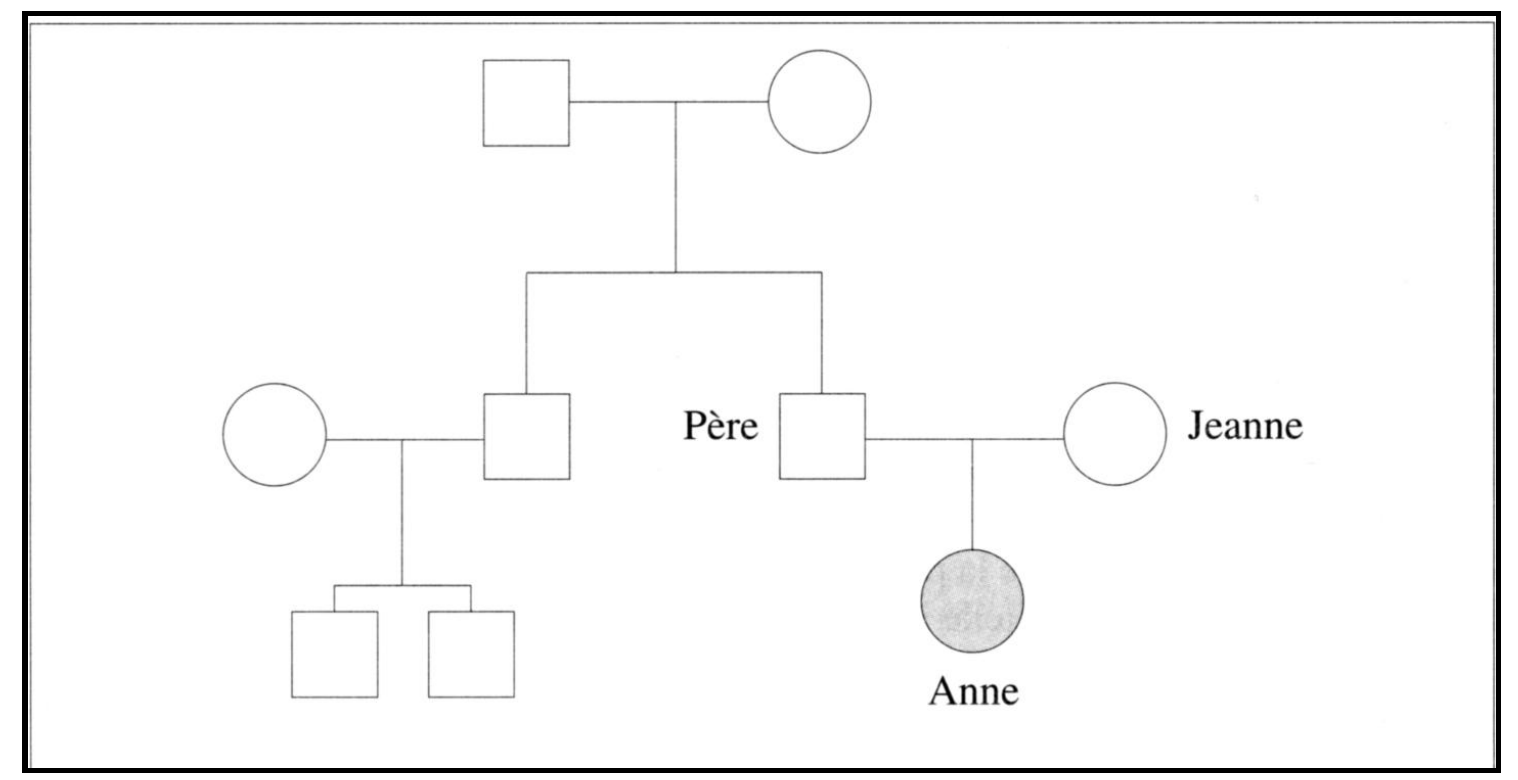

Figure 3. Famille du père d'Anne dans la configuration 3. Vers une communauté clanique

Et pourtant, cette unité si visible est un phénomène qui émerge d'un puissant antagonisme caché qui organise la famille. Le frère aîné et sa belle réussite constituent un premier pôle qui soutient les croyances familiales. L'arrière-grand-père était géomètre, le grand-père conducteur de travaux et le diplôme d'ingénieur de son fils aîné renforce le statut social de la famille. Ce fils aîné est socialement valorisé, on ne parle que des valeurs qu'il soutient. Le fils cadet et Anne constituent le deuxième pôle. Les deux pôles ne sont pas en rivalité directe : le père d'Anne ayant « raté » ses études, c'est plutôt par le malheur qui lui est arrivé - avoir une fille malade - qu'il va lutter sous cape avec son frère aîné. Mais qui des deux frères est le plus à même d'unifier la famille ? L'aîné et sa brillante réussite professionnelle qui fait la fierté de tous et qui, en s'expatriant et en imposant une si grande distance physique avec les siens augmente l'attention qui est portée sur lui? Ou le cadet qui réussit à rassembler toute sa famille (grand-mère, oncles et tantes et même son frère) autour de la «pauvre » Anne et qui consacre beaucoup de temps à sa mère en s'occupant d'elle quotidiennement? Voilà l'enjeu. Un enjeu très caché car le cadet est fier de la réussite de son frère, lequel rend visite, à chacun de ses voyages, à son cadet et surtout à sa nièce Anne, en plaignant son frère. Si chaque frère avait conscience de sa place dans le processus qu'il anime, chacun exprimerait sa conviction que c'est lui seul qui réalise l'unité familiale ou la soudure communautaire, alors qu'en réalité ce qui fait soudure et unifie la famille, c'est l'antagonisme caché entre les deux frères.

Ce processus, qui situe précisément la place importante du père d'Anne dans la stratégie mise au point par sa famille d'origine, rend davantage problématique la position d'Anne qui prend, dans la famille de son père, une fonction importante sans laquelle l'équilibre des forces antagonistes serait rompu. Sans en dire davantage, on comprend que la question de la psychothérapie d'Anne devrait prendre une nouvelle tournure dont il semble difficile à présent de faire l'économie.

\section{Vers une communauté clanique (figure 3)}


Le père d'Anne pourrait être membre d'une communauté qui cultive fièrement le sens de la famille en faisant prospérer son héritage. Que celui-ci soit matériel - comme une usine, une entreprise, un magasin ou une ferme -, qu'il soit professionnel - on est médecin de père en fils, avocat, ingénieur, cheminot, politicien, professeur, employé, etc. -, chaque fois, on rencontre l'idée d'un héritage reçu à faire fructifier et à transmettre à la génération suivante. Ce sont les croyances et les représentations que la famille se fait d'elle-même qui sont ainsi affirmées : les valeurs traditionnelles, l'argent, la religion, la réputation, le travail, la famille, la patrie, le devoir, etc. L'éthique officielle - le code normatif - est en accord avec les croyances ; tous affirment qu'il faut faire fructifier l'héritage reçu et ils s'y emploient concrètement.

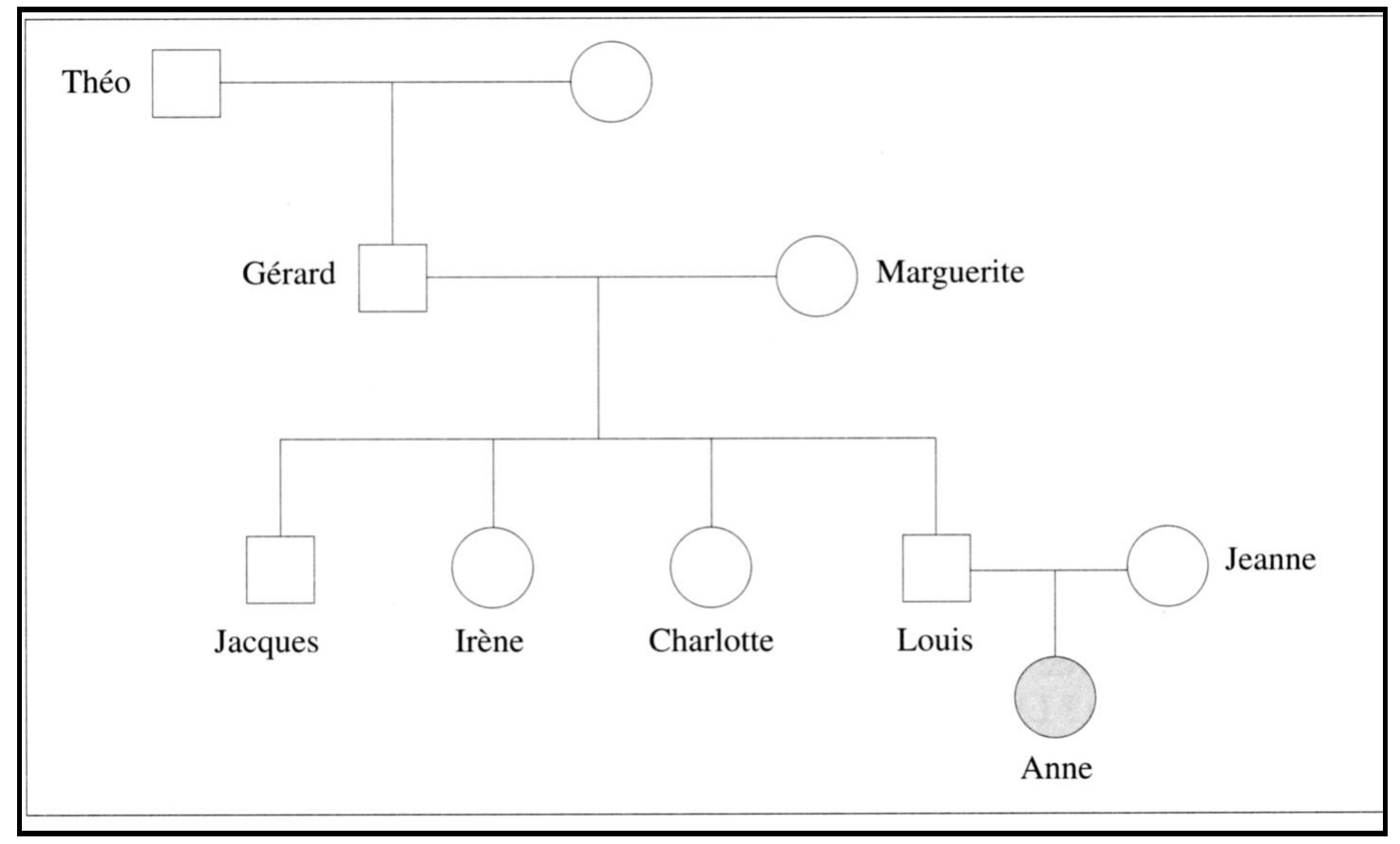

Figure 3: Famille du père d'Anne dans la configuration 3. Vers une communauté clanique.

Voilà à peu près le contenu du discours familial lors d'une première rencontre thérapeutique. Présentons les membres d'une de ces familles où pourrait se rencontrer, à nouveau, le père d'Anne:

- Gérard, le père de famille, est entrepreneur en bâtiments. Il est responsable des corps de métier qui interviennent dans la construction d'un immeuble et il traite directement avec le maître d'oeuvre et l'architecte. Son père, Théo, était déjà dans la partie, mais plus modestement, il était entrepreneur en maçonnerie; c'est le père de Théo, simple maçon, qui l'a initié au métier. Les affaires ont prospéré si bien que Gérard a développé l'entreprise.

- Margueritte, l'épouse de Gérard, a épaulé son mari, s'est occupée de la gestion de la maison et a élevé quatre enfants. Très tôt, elle a souffert de polyarthrite.

- Jacques, l'aîné des enfants, est ingénieur civil et travaille avec son père. Il compte développer un département de génie civil : construction de routes, ponts, autoroutes, lignes de chemin de fer TGV.

- Irène, l'une des filles, est diplômée en administration des affaires, elle est respon- 
sable du secrétariat et de la comptabilité de l'entreprise familiale.

- $\quad$ Charlotte, une autre fille, a entrepris des études de psychologie qu'elle a abandonnées, elle s'est inscrite ensuite en histoire mais sans succès. En fait, son père voulait qu'elle obtienne un diplôme universitaire. Charlotte montrant beaucoup d'aptitudes artistiques, après de multiples péripéties, son père lui ouvre une galerie d'art. Ses nombreuses aventures sentimentales défraient la chronique familiale. Chaque fois qu'elle est amoureuse, elle disparaît de l'horizon mais dès la rupture, elle se réfugie auprès des siens qu'elle a peine à quitter malgré les nombreuses critiques qui lui sont faites: son travail à la galerie n'est pas vraiment un travail, elle est incapable de se trouver un mari et de fonder une famille comme les autres, etc.

- Louis est le benjamin, il est le père d'Anne. Suite à une maladie, il a arrêté ses études d'ingénieur et s'est inscrit à un régendat littéraire qu'il a réussi. Il a enseigné quelques années, mais sa mauvaise santé et les chahuts qu'il subissait lui ont fait quitter l'enseignement. Son père lui a alors trouvé une place dans l'entreprise, il travaille au secrétariat. Mais devant le peu de rentabilité de son action et surtout les erreurs qu'il commet, son père lui confie de moins en moins de responsabilité; aujourd'hui, il fait quasi ce qu'il veut dans son bureau et, aux dernières nouvelles, il confectionne des bateaux dans des bouteilles (sic). Quand il n'est pas à son bureau, on le trouve près de sa mère souffrante ou près de sa soeur qu'il aide à monter ses expositions de peinture.

Lors de la première séance, la famille parle d'une seule voix, aucune différence n'apparait, aucune critique du mode de vie familial n'affleure qui distinguerait un membre d'un autre; les informations concernant la vie de chacun d'eux nous seront données au fur et à mesure des séances. La différence, nous la repérons quand nous examinons la manière dont ils vivent le monde du sentir.

Un premier sous-système, que nous appellerons « dominant », est composé de Gérard, de Jacques et d'Irène. Ce sous-groupe soutient très activement les valeurs dominantes de la famille, ses membres s'activent à conduire correctement l'entreprise, à la faire prospérer. Ils vivent surtout dans la production, l'efficacité, le rendement. Mais aussi, ils sont les premiers à participer aux activités sociales et mondaines qui concrétisent leur adhésion au monde bourgeois des bien-pensants de la ville. Ils créent ainsi une puissante unité familiale autour du projet économique et social de la famille ; ils sont peu sensibles au monde du sentir, où ils semblent vivre davantage dans la distance.

Un deuxième sous-système, que nous appellerons « contestant caché », est composé de Margueritte, Charlotte et Louis à qui il faut adjoindre Anne. Tout en soutenant verbalement le sous-système «dominant», ces membres réintroduisent une autre forme d'unité à partir de leurs comportements analogiques et de leur manière particulière d'animer le monde du sentir. Ce sous-système active une proximité du contact et, au-delà d'elle, il «force» une unité familiale d'un tout autre ordre que celle engendrée par le premier sous-système beaucoup plus attentif au paraître et à l'avoir. La mère souffre dans son corps et, à ce titre, elle se sent proche de sa petite fille Anne. Charlotte, en échouant à l'université, s'oppose aux valeurs de son père et en développe d'autres liées à l'art et à l'esthétique, liées aussi à la vie de Bohème et aux passions amoureuses. Mais ces valeurs ne sont pas proclamées, elles sont plutôt vécues et mises en actes. A chaque problème qu'elle rencontre, Charlotte retourne vivre chez les siens où elle demande de l'argent à son père tout en se faisant câliner comme un enfant par sa mère. Ces retrouvailles sont parfois 
orageuses, le père s'arrache les cheveux, sermonne sa fille et la conjure de changer son mode de vie, mais il continue à éponger le déficit de la galerie d'art. Lorsque la mère intervient dans le conflit, ce sont les rares moments où le couple exprime ouvertement un bref désaccord. Quant à Louis, tous semblent le protéger. Sa santé fragile et le peu de compétence qu'il manifeste pour les affaires sont autant d'excuses pour lui «pardonner» son manque de productivité. Pourtant, il tient à sa place comme à la prunelle de ses yeux, jamais il ne manquerait une journée de travail; même s'il est mal portant, il vient se soigner près de sa mère à qui il fait parfois la lecture de poèmes qu'il a écrits en secret. Le monde du sentir est aussi important pour Louis, car il est très sensible aux douleurs de sa mère, il souffre lui-même, il ne produit quasi rien, si ce n'est la fabrication de bateaux dans des bouteilles et l'écriture de poèmes. Ce sous-système a la capacité de mobiliser l'ensemble de la famille autour de lui: la maladie de la mère réunit tout le monde lorsque sa polyarthrite la fait souffrir davantage ; les frasques de Charlotte attirent l'attention de tous, spécialement l'attention du sous-système dominant qui sent que ses valeurs sont bafouées. Le style de vie de Louis, peu compatible avec la gestion d'une entreprise, ferait sourire s'il n'avait pas une enfant handicapée. Anne vient régulièrement dans l'entreprise avec son père et personne ne peut résister à recréer autour d'elle et de son père une proximité familiale qui semble être une séquence, un arrêt sur image, d'une situation familiale tellement tournée vers les affaires qu'elle en oublie de vivre et de partager ensemble des moments gratuits de convivialité.

Les deux sous-systèmes - particulièrement malaisés à distinguer - sont effectivement dans un rapport antagoniste bien que le caractère antagoniste soit caché par la manière dont chaque sous-système réalise l'unité familiale. Les membres du sous-système « dominant » ont le sentiment profond qu'ils réalisent l'unité familiale par la bonne gestion de l'entreprise. Celle-ci est le véritable ciment unificateur de la famille et, lors des premiers entretiens, toute la famille est en accord avec l'importance de l'unité familiale autour des valeurs traditionnelles où le paraître, la position sociale et l'argent occupent l'essentiel de la place au détriment de valeurs moins brillantes comme celles du coeur, de la souffrance et de l'esthétique dont personne ne parle. Ces dernières valeurs animent pourtant le soussystème « contestant caché » à partir de quoi s'élabore une autre forme d'unité, construite tout différemment que la première et auquel toute la famille participe également. Tandis que l'unité autour de l'entreprise implique le mythe familial et le code normatif qui met concrètement en scène le mythe, l'unité autour de la souffrance et de l'esthétique impliquent des valeurs que le mythe fait taire: celles issues davantage de l'ambiance. Si chaque sous-système a la conviction de réaliser à lui seul la véritable unité familiale, en réalité, c'est la conjonction des deux manières de faire unité - à force égale - qui donne vie à la communauté.

\section{Un clan familial (figure 4)}

Le quatrième père hypothétique d'Anne pourrait être Gaston, père de Fanette, dont nous allons décrire la famille. La première fois que nous avons pu nous faire une idée de l'organisation d'une famille extrêmement communautaire date de quasi trente ans (2). Une famille veut placer sa fille Fanette, une adolescente autiste, dans l'institution où nous travaillons. En attendant une place disponible, nous recevons régulièrement la famille en vue d'établir une collaboration entre elle, la jeune fille et l'institution. Le jour du premier 
rendez-vous, le père, Gaston, la mère, Dorothée, et la jeune fille, Fanette, sont déjà installés dans la salle de consultation, le père et la fille se sont assis dans le même fauteuil sans accoudoir, tandis que la mère, à trois fauteuils de distance, fait face à ce couple étrange. Les fauteuils ne sont pas larges et le père comme la fille étant de fortes personnes, ils occupent chacun la moitié du fauteuil et doivent, pour ne pas tomber, s'accrocher l'un à l'autre comme le feraient des amants.

Voici l'histoire de Gaston. Peu après sa naissance, ses parents s'installent en Campine ${ }^{13}$ avec leurs dix enfants dans une ferme isolée au milieu d'une clairière entourée de bois.

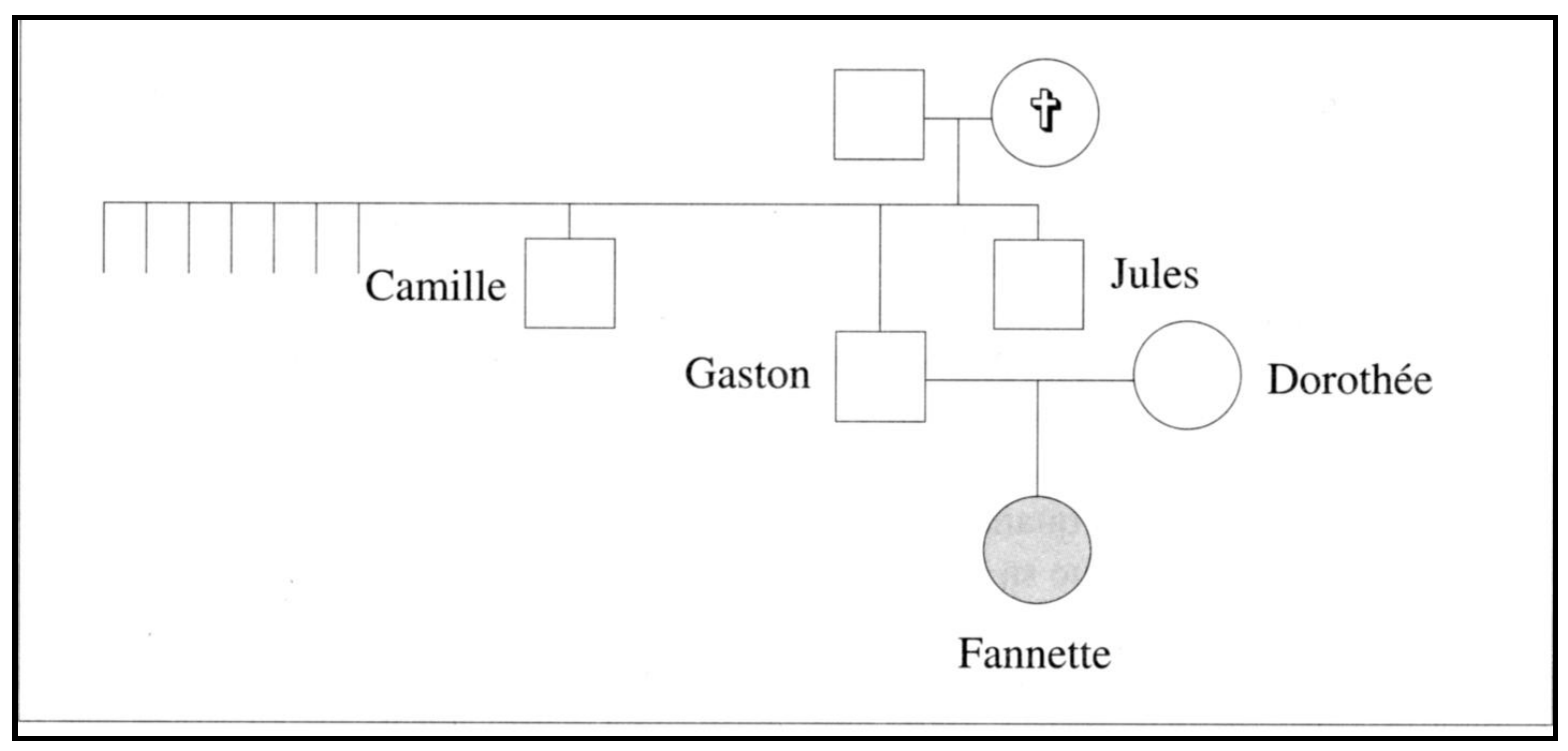

Figure 4. Famille du père de Fanette. Un clan familial.

Tout à fait repliée sur elle-même, la famille vit en complète autarcie. Comme dans un clan, chaque membre est soumis au contrôle des autres et l'union dans le travail est leur credo. Les valeurs chrétiennes sont étroitement liées au respect des valeurs ancestrales : travail, famille, honneur, etc., et contribuent à la consolidation du clan. La vie à la campagne est dure et la souffrance est le lot commun : nombreux accidents à la ferme, accidents d'accouchement et souffrances physiques ponctuent le labeur. Entre autres, Gaston a toujours connu sa mère impotente qu'il fallait soigner et entourer. Si le travail est garant de la prospérité matérielle, la souffrance qui l'accompagne assure la sainteté, au point que le travail et la souffrance sont des valeurs complémentaires qui définissent le clan. C'est dans un tel contexte que grandit Gaston ; il n'a qu'un désir, faire de sa vie l'accomplissement de la tradition familiale.

Gaston termine sa scolarité primaire quand sa mère meurt. Peu après, la soeur aînée qui avait pris le rôle de mère meurt en couche et Jules, le fils cadet, prend en charge la gestion du ménage. A cette époque, les frères et soeurs aînés sont mariés ; les femmes ont épousé des fermiers des alentours, tandis que le père a acheté lui-même des fermes à la périphérie de son «domaine » pour ses fils. Il reste à la ferme paternelle les plus jeunes

${ }^{13}$ La Campine est une région de Belgique au Nord de Liège. 
enfants: Camille, Gaston et Jules. Camille est l'aîné du sous-groupe, il devient un fermier averti et son esprit d'entreprise autant que la sûreté de son jugement le désigne comme le successeur du père. Jules restera célibataire, il demeurera là et se dévouera à son père comme il s'était dévoué à sa mère. Quant à Gaston, à peine termine-t-il l'école primaire que son père lui propose d'entreprendre des études techniques. L'école est à 40 kilomètres et l'enfant devra y prendre pension. Malgré la grande autorité du père, Gaston refuse la proposition. Le père insiste: «Tu es intelligent et tu regretteras plus tard de ne pas avoir fait des études d'autant que je constate que tu n'es pas doué pour les travaux de la ferme et que tu ne deviendras jamais un aussi bon fermier que tes frères ». Pris de terreur à l'idée de quitter le clan, Gaston refuse à nouveau : " On est bien ensemble, je ne veux pas quitter ma famille ». Gaston reste, jusqu'à son mariage, avec son père et ses frères et peu à peu il réalise la prédiction de son père en devenant le médiocre fermier qu'il est demeuré, selon ses dires, toute sa vie.

Durant son adolescence, il ressent douloureusement l'écartement géographique qu'il devra accepter lorsqu'il se mariera. Il le vit surtout lorsque Camille devient le nouveau patron car il doit se rendre à l'évidence que le creuset familial ne lui laissera plus sa place. A son mariage, il sait qu'il doit s'exiler sur des terres lointaines, à $10 \mathrm{~km}$ de Camille (sic), où il contribuera mal à la prospérité matérielle du clan.

Gaston entre en crise quand son père le définit comme incapable de poursuivre l'oeuvre familiale, alors que sa culture lui impose, depuis toujours, de fortifier le clan par son travail et par le développement de compétences professionnelles. Il faut souligner que l'organisation du clan empêche radicalement qu'une lutte ouverte s'installe entre lui et Camille, lutte dans laquelle Gaston aurait pu rivaliser sur le même «terrain » que son frère. Dans ces circonstances, Gaston est en bute à deux contraintes incompatibles, l'une en provenance du milieu (contribuer à la prospérité du clan), l'autre directement de son père (tu ne seras jamais un bon fermier). Quoi qu'il fasse, Gaston est en situation de choix impossible. L'un comme l'autre le rend indigne de confiance soit vis-à-vis du milieu culturel, s'il obéit à son père, soit vis-àvis de son père s'il devient un bon fermier. Ce choix impossible le rend malade. Il déprime, se plaint, devient inactif, souffre de maux étranges, se referme en lui-même, et sa manière d'être appelle une assistance continue. Ses symptômes ont comme effet d'inquiéter tous les membres de la famille qui, à tour de rôle, ou en groupe visitent le malade, s'inquiètent de son sort et font ce qu'ils ont toujours fait lorsqu'un de leur membre souffrait. A ce moment, tout se passe comme si l'ensemble des valeurs complémentaires «travail/souffrance » se clivait tout à coup dans l'esprit de Gaston et que chacune prenait son indépendance: Camille soutient l'unité par le travail et la prospérité matérielle et Gaston par la réunification «spirituelle » que sa maladie impose à l'ensemble de la famille. Cette imposition que Gaston fait constamment peser sur l'ensemble de la famille le sort de son propre dilemme d'être ou non digne de confiance : tout en obéissant à la prévision du père (être un médiocre fermier), il se soumet aussi à la contrainte de son milieu culturel (fortifier le clan) lorsqu'il opère un nouveau recadrage qui place la souffrance comme le meilleur moyen de consolider le clan.

La nouvelle organisation qui fait suite à la crise de Gaston situe Camille dans le rôle de « dominant », celui-ci impose à tous l'idée de clan par le thème unique du travail et de la prospérité, tandis que Gaston prend le rôle du "contestant caché » lorsque, tout en acceptant le thème imposé par son frère et son départ inéluctable de la ferme paternelle, il 
impose constamment à tous l'idée d'un clan spirituel et relationnel qui se reforme autour de sa personne souffrante. Cette organisation se perpétue et devient un moteur important dans le maintien des comportements autistiques de Fanette, car, au moment où celle-ci est diagnostiquée « autiste », Gaston se débarrasse comme par miracle de ses symptômes et c'est par Fanette que l'antagonisme avec Camille se poursuit.

C'était la première fois que nous rencontrions une organisation aussi soudée, et il fut très difficile d'accéder à la différenciation telle que nous venons de la décrire. Tout au long des séances, Gaston ne cessait de louanger tantôt son père, tantôt son frère qui avait toutes les qualités possibles, tandis que lui n'était qu'un mauvais fermier. Pour nous en convaincre, il énumérait toutes les erreurs qu'il commettait en prenant sa femme à témoin : les vaches crevaient parce qu'il n'avait pas décelé à temps les signes d'une maladie que tout fermier, ajoute-il, doit voir afin de faire soigner la bête à temps. Et les séances se succédaient en tournant inlassablement autour des mêmes thèmes au point que nous devenions agacés : par de nombreux indices, il était évident qu'une lutte sourde et quasi sans merci opposait Gaston à son frère Camille. Aussi, un jour où Gaston s'enflammait encore pour glorifier le travail de son frère, nous avons brutalement mis en question sa fonction dont il ne parlait quasi jamais, celle de réunir à sa façon la famille: nous avons affirmé «Au fond, vous ne faites rien pour votre famille, Camille fait tout» et nous avons développé le thème avec tant de conviction que Gaston s'est levé, fâché, et qu'il a déclaré d'une traite «Je suis plus fort avec Fanette que Camille avec ses trente têtes de bétail! ». Puis il s'est rassis, éberlué par ce qu'il venait de révéler. Nous aussi étions éberlués car pour la première fois s'exprimait ouvertement le puissant antagonisme organisateur du clan dans lequel la maladie de Fanette occupait une place prépondérante.

\section{En conclusion}

Les quatre exemples ont illustré différentes soudures communautaires : du fils soutien de veuve à Gaston, nous avons évoqué des situations de plus en plus contraignantes dans lesquelles le père de l'enfant malade - qu'il s'agisse d'Anne ou de Fanette - maintient fermement une fonction essentielle dans un processus communautaire très particulier, en s'aidant de son enfant malade, devenu en quelque sorte son bras armé, dans le combat qu'il mène, sans vraiment le savoir, dans sa famille d'origine.

Depuis la parution de son livre «La Méthode », E. Morin (7) nous a familiarisés à l'idée d'antagonisme organisateur qui permet de mettre en système deux forces radicalement opposées, autrement dit de saisir l'association qu'elles forment. Le soleil émerge d'une opposition entre deux forces: les explosions thermonucléaires sont des forces centrifuges, tandis que la gravitation du soleil sur lui-même produit une force centripète. Lorsqu'une force l'emporte sur l'autre, l'étoile a fini sa carrière, elle devient soit une supernova lorsque les forces centrifuges dominent définitivement, soit un trou noir lorsque la force centripète l'emporte. Quand il s'agit des sciences humaines, et particulièrement de la communauté devenue rigide, cet antagonisme a la particularité de sembler disparaître au profit d'une seule manière de faire l'unité, c'est du moins ainsi que la communauté se présente: sans faille, ronde, impénétrable et à l'unisson. La découverte de la différence, indispensable à l'existence de toute organisation, qu'elle soit physique ou humaine, se découvre à travers l'ambiance et ses qualités où, à l'abri des regards, elle agit avec autant 
de puissance que le discours dominant, en réintroduisant la différence tout en la dissimulant aux yeux des profanes. Le modèle que nous présentons est à « géométrie variable », c'est-à-dire qu'il fonctionne autant pour des communautés dynamiques, celles qui ne produisent pas de pathologie, que pour des communautés claniques où le maintien de l'équilibre des forces fait appel à la puissance des comportements symptomatiques. En d'autres termes, une organisation communautaire dynamique peut, au gré des circonstances, rompre l'équilibre de ses forces et, comme le soleil, disparaître en tant que communauté organisée. On assiste alors à son morcellement et à l'éparpillement de ses membres ; par exemple, lors du décès d'un aïeul fédérateur. Mais dans certains cas ce morcellement est impossible pour des raisons de culture familiale à rechercher; la communauté a alors la capacité de produire une rééquilibration de ses forces par l'émergence de comportements symptomatiques qui assurent une nouvelle organisation et une nouvelle vie à la communauté. Notre démarche associe le modèle de la communauté rigide à celui de la triple contrainte, et tous deux révèlent le bien-fondé culturel de la situation, les raisons ou encore la logique sous-jacente qui permet à la communauté de survivre au prix de l'émergence d'un ou de plusieurs patients désignés. Il reste, avant d'aborder la question du traitement, à revenir à la mère d'Anne en recherchant son processus d'individuation et en comprenant la place qu'elle a occupée et qu'elle occupe encore dans sa famille d'origine, corrélativement comprendre aussi la formation du couple, afin de saisir en quoi son processus d'individuation la met en prise directe avec le double lien qu'elle maintient avec sa fille.

\section{BIBLIOGRAPHIE}

1. Bateson G., Bateson M.C. (1989) : La Peur des Anges. Vers une épistémologie du sacré, Seuil, Paris.

2. Dessoy E. (1985) : Psychiatrie, autisme infantile et société. Autisme et organisation familiale, Cabay, coll. Perspective sur l'homme, Louvain-la-Neuve, 7, pp. 77-105.

3. Dessoy E. (1993) : Le milieu humain I : de l'intérêt du concept en psychothérapie institutionnelle et en approche systémique. Thérapie familiale, 14 (4), pp. 311-330.

4. Dessoy E., Compernol C., Pauss V. (1994): Le milieu humain II: étude de cas. L'impact de l'enfant psychotique sur le milieu familial et le milieu institutionnel : une collaboration entre famille et institution. Thérapie familiale, 15 (1), pp. 79-90.

5. Dessoy E. (1997) : Ambiance et double lien. Thérapie Familiale, 18 (4), pp. 367-385.

6. Dessoy E. (1998) : Croyances et double lien. Thérapie Familiale, 19 (4), pp. 367-396.

7. Morin E. (1977) : La méthode, T. I: La nature de la nature, Seuil, Paris. 